\title{
Observation of a molecular muonium polaron and its application to probing magnetic and electronic states
}

\author{
M. Rogers $\odot,{ }^{1, *}$ T. Prokscha $\odot,{ }^{2}$ G. Teobaldi $\odot,{ }^{3,4,5}$ Leandro Liborio $\odot,{ }^{3}$ S. Sturniolo $\odot,{ }^{3}$ E. Poli $\odot,{ }^{3}$ D. Jochym $\odot,{ }^{3}$ \\ R. Stewart, ${ }^{6, \dagger}$ M. Flokstra, ${ }^{6}$ S. Lee,${ }^{6}$ M. Ali, ${ }^{1}$ B. J. Hickey, ${ }^{1}$ T. Moorsom,,${ }^{1,}$ and O. Cespedes ${ }^{1, \S}$ \\ ${ }^{1}$ School of Physics and Astronomy, University of Leeds, Leeds LS2 9JT, United Kingdom \\ ${ }^{2}$ Laboratory for Muon Spin Spectroscopy, Paul Scherrer Institute, 5232 Villigen, Switzerland \\ ${ }^{3}$ Scientific Computing Department, Science \& Technology Facilities Council, Rutherford Appleton Laboratory, \\ Didcot OX11 0QX. United Kingdom \\ ${ }^{4}$ Stephenson Institute for Renewable Energy, Department of Chemistry, University of Liverpool, Liverpool L69 3BX, United Kingdom \\ ${ }^{5}$ School of Chemistry, University of Southampton, Highfield, Southampton SO17 1BJ, United Kingdom \\ ${ }^{6}$ School of Physics and Astronomy, SUPA, University of St Andrews, St Andrews KY16 9SS, United Kingdom
}

(Received 30 January 2021; revised 3 June 2021; accepted 21 July 2021; published 17 August 2021)

\begin{abstract}
Muonium is a combination of first- and second-generation matter formed by the electrostatic interaction between an electron and an antimuon $\left(\mu^{+}\right)$. Although a well-known physical system, their ability to form collective excitations in molecules had not been observed. Here, we give evidence for the detection of a muonium state that propagates in a molecular semiconductor lattice via thermally activated dynamics: a muonium polaron. By measuring the temperature dependence of the depolarization of the muonium state in $\mathrm{C}_{60}$, we observe a thermal narrowing of the hyperfine distribution that we attribute to the dynamics of the muonium between molecular sites. As a result of the time scale for muonium decay, the energies involved, charge and spin selectivity, this quasiparticle is a widely applicable experimental tool. It is an excellent probe of emerging electronic, dynamic, and magnetic states at interfaces and in low dimensional systems, where direct spatial probing is an experimental challenge owing to the buried interface, nanoscale elements providing the functionality localization and small magnitude of the effects.
\end{abstract}

DOI: 10.1103/PhysRevB.104.064429

\section{INTRODUCTION}

The ability to map in a multilayered thin film electronic, vibrational and spin order in thin films is key to many chemical, engineering and physical applications, in particular for compounds involving nanocarbon and molecular materials $[1,2]$. Yet, the direct spatial probing of spin ordering and electronic properties is an experimental challenge, owing to the nm-scale localization and small magnitude of the effects. Magnetic proximity, spin accumulation and spontaneous spin order in non-magnetic metals can lead to localized magnetic phenomena that are at the lower limit of sensitivity of laboratory-based and synchrotron magnetometry techniques [3-6]. Mapping charge distribution is also an essential challenge to, e.g., solid-state (thin-film) batteries and fullerene hybrid photovoltaics $[7,8]$. In the past, bulk muon spin rotation $(\mu \mathrm{SR})$ has been used extensively to study various properties

\footnotetext{
*M.D.Rogers@leeds.ac.uk
}

${ }^{\dagger}$ Current address: Laboratory for Mesoscopic Systems, Department of Materials, ETH Zurich, 8093 Zurich, Switzerland and Laboratory for Multiscale Materials Experiments, Paul Scherrer Institute, 5232 Villigen PSI, Switzerland.

${ }^{\ddagger}$ Current address: Kelvin Building; University of Glasgow, Glasgow, G12 8QQ, United Kingdom.

§O.Cespedes@leeds.ac.uk of the fullerenes including, but not limited to: rotational dynamics $[9,10]$, superconducting properties of the alkali-doped fullerene complexes [11], and spin ordering in the TDAE- $\mathrm{C}_{60}$ charge-transfer complex [12].

Low-energy muon spin rotation (LE- $\mu \mathrm{SR}$ ) is also a powerful tool in characterizing interfacial and thin film spin phenomena for a multitude of systems [13-16]. Here, LE$\mu \mathrm{SR}$ is used first to show the presence of low frequency endohedral muonium in $\mathrm{C}_{60}$, coupled to the vibrational states of the molecule at all temperatures. The depolarization of this state is linked to the dynamics of the molecular cages, as two distinct dynamical phases can be seen in the temperature dependence of this state. This muonium polaron is a highly sensitive probe to local electronic, vibrational and spin states. Here, we use it to probe changes in electron density at metal interfaces and emergent magnetism down to $\sim 10$ s of $\mu \mathrm{T}$ via Zeeman splitting of the hyperfine interaction. The principle for this probing technique is shown in Fig. 1(a).

\section{METHODS}

For all measured samples, metal films were deposited by DC magnetron sputtering and $\mathrm{C}_{60}$ films were sublimated in a high vacuum system with a $10^{-8} \mathrm{mbar}$ base pressure. Sputtering was undertaken in a $3.3 \times 10^{-3}$ mbar Ar atmosphere at ambient temperature unless otherwise specified. The growth rate of each material was calibrated against samples 

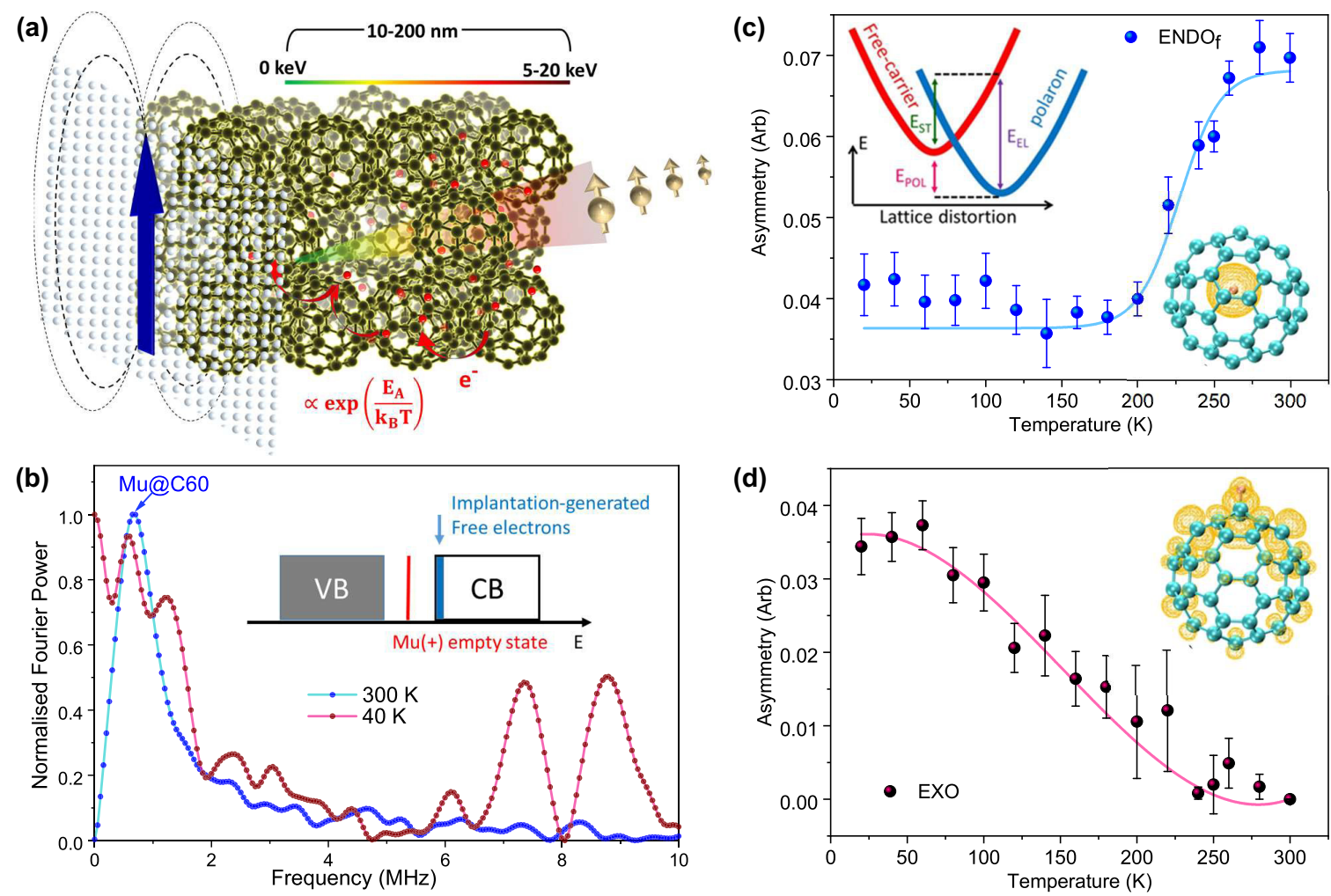

FIG. 1. (a) Schematic of the $\mathrm{ENDO}_{\mathrm{f}}$ formation via ionization, charge hopping, and trapping then coupled with molecular rotations. Its oscillation frequency is sensitive to, e.g., the electron transfer and emergent magnetism at interfaces. (b) Fourier transform of the time domain asymmetry data at $\mathrm{ZF}$ for a 210 -nm- thick amorphous $\mathrm{C}_{60}$ film on a thermally oxidized Si substrate. The EXO muonium at 1.2, 7.4, and 8.6 MHz is observable at $40 \mathrm{~K}$. At $300 \mathrm{~K}$, only the $\mathrm{ENDO}_{\mathrm{f}}$ muonium is observed. Inset: $(\sim \mathrm{keV})$ muon-beam generated free carriers remain delocalized in the conduction band (CB) of the $\mathrm{C}_{60}$ film, leaving the e-acceptor state of the $\mu^{+}$empty. (c) The temperature dependence of the asymmetry attributed to the low-frequency $\mathrm{ENDO}_{\mathrm{f}}$ together with the calculated spin-density using the PBE exchange-correlation (XC) functional (yellow: $10^{-6} \mu_{\mathrm{B}} \AA^{-3}$ ). A semiempirical model has been used to fit the temperature dependence leading to an activation energy (equivalent to $-\mathrm{E}_{\mathrm{POL}}$ ) for $\mathrm{ENDO}_{\mathrm{f}}$ of $\mathrm{E}_{\mathrm{A}}=300 \pm 100 \mathrm{meV}$ [30]. (d) Temperature dependence of the EXO signal fraction, also with the calculated PBE spin-density (yellow: $10^{-6} \mu_{\mathrm{B}} \AA^{-3}$ ). The EXO state becomes more apparent as we cool the system through the glass transition and the molecular rotations are frozen.

grown in the same vacuum cycle whose thickness was determined via the fitting of Kiessig fringes produced by x-ray reflectivity measurements.

LE- $\mu \mathrm{SR}$ measurements were performed on the $\mu \mathrm{E} 4$ beamline at the Paul Scherrer Institute, where a moderator technique allows for the implantation energy of nearly $100 \%$ spin polarized epithermal muons to be tuned via an acceleration electric field $[17,18]$. Thus, low-energy muons allow for the depth profile of magnetic texture to be obtained. The energies required to probe the $\mathrm{Cu} / \mathrm{C}_{60}$ were determined through the use of Monte Carlo simulations performed within TRIM.SP software [19]. The muon is an unstable spin $1 / 2$ particle with charge $+e$ and a lifetime of $\tau_{\mu} \sim 2.2 \mu \mathrm{s}$. Following implantation, the muon will rapidly thermalize. In a system which lacks sufficient carrier concentration to screen the $\mu^{+}$charge, such as insulating and semiconducting systems, the muon is expected to generate a hydrogenlike bound state known as muonium $\left(\mathrm{Mu}=\mu^{+} e^{-}\right)$. In a metallic system, one would observe the Larmour spin precession of the $\mu^{+}$particle about a local field $B_{\text {loc }}$. In a system where the muonium formation dominates the signal, we directly probe transitions between spin configurations of the $\mu^{+}$and $e^{-}$spin, governed by the Hamiltonian which describes the hyperfine interaction. This is given, in terms of angular frequency, as

$$
\frac{H_{H F}}{\hbar}=-\gamma_{\mu} \boldsymbol{S}^{\mu} \cdot \boldsymbol{B}+\gamma_{e} \boldsymbol{S}^{e} \cdot \boldsymbol{B}+\boldsymbol{S}^{\mu} \cdot A \cdot \boldsymbol{S}^{e},
$$

where $\mathbf{S}^{\mu}$ and $\mathbf{S}^{e}$ are the respective muon and electron spin operators, $\gamma_{\mu}$ and $\gamma_{e}$ are the gyromagnetic ratios for the electron and muon, $A$ is the hyperfine coupling tensor, and $\mathbf{B}$ is the magnetic field $[20,35]$. For an isotropic hyperfine tensor $\left(A_{x x}=A_{y y}=A_{z z}\right)$, two states exist in zero-field: a singlet and a degenerate triplet state. The application of a magnetic field lifts the degeneracy of the triplet state and then transitions between energy levels can be observed as an oscillation frequency $v_{i j}$ which is equal to the splitting between energy levels $\left(E_{i}-E_{j}\right) / \hbar$. The splitting between the $\mathrm{ZF}$ states is equal to the hyperfine coupling constant $A_{H}=$ $2 \pi \times 4463 \mathrm{MHz}$. This frequency would be unobservable in a typical $\mu \mathrm{SR}$ experiment. Therefore, it is expected that no oscillation should be observed in the isotropic hyperfine case. If, however, some axial anisotropy is introduced into the hyperfine tensor, the degeneracy of the triplet state is lifted and a single frequency can now be expected in the absence of magnetic field. The $\mu \mathrm{SR}$ data were analyzed using the program MUSRFIT [21,41]. 


\section{OBSERVATION OF THE FLOATING ENDOHEDRAL MUONIUM POLARON IN C 60 (ENDO $\left.{ }_{\mathrm{f}}\right)$}

The zero-field (ZF) muonium oscillation data for an amorphous, thick $(\sim 200 \mathrm{~nm}) \mathrm{C}_{60}$ film has been fitted using a multiple frequency model, where an individual precession frequency $v_{i}$ has a contribution to the muon polarization, $P$ $(t)$, given by

$$
P(t)=\sum_{i=1}^{4} A_{i} \cos \left(2 \pi v_{i} t\right) e^{\left(-\lambda_{i} t\right)},
$$

where $\lambda_{\mathrm{i}}$ is the depolarization rate of the oscillating muonium. The asymmetry, $A_{i}$, is then the fraction of signal attributed to a given oscillation; see the Supplemental Material [22].

At room temperature, $\mathrm{C}_{60}$ crystals form a face-centeredcubic (fcc) structure where the individual molecules are orientationally disordered and freely rotate between degenerate configurations via a ratcheting mechanism [23]. Below $260 \mathrm{~K}, \mathrm{C}_{60}$ molecules lose two rotational degrees of freedom. Due to inequivalent molecular orientations on the Bravais sites of the fcc lattice, crystalline $\mathrm{C}_{60}$ undergoes a first-order phase transition to a simple cubic structure. Below $\sim 120 \mathrm{~K}$, the time scale of the molecular rotation $\left(\tau_{\mathrm{r}}\right)$ is longer than the measurement time scale for $\mu \mathrm{SR}\left(\tau_{\mathrm{m}} \sim 2.2 \mu \mathrm{s}\right)$, and the rotational degrees of freedom appear frozen [24,25]. As shown in Fig. 1(b), at low temperatures, oscillations at 1.2, 7.4, and 8.6 MHz are visible. These frequencies are well understood and are attributed to the completely anisotropic hyperfine interaction of the exohedral radical muonium state (EXO) [26,27], but can only be detected when $\tau_{\mathrm{r}}>\tau_{\mathrm{m}}$.

In addition to the $\mathrm{MuC}_{60}$ radical precession, we observe an additional low-frequency precession that persists up to room temperature. This room-temperature oscillation has not been reported in the various studies of bulk crystalline $\mathrm{C}_{60}$ $[27,28]$. However, it is seen in all the thin fullerene films grown throughout this study, with stronger signals in the more disordered films. This high temperature muonium state has been shown to be sensitive to magnetic surface states $[3,4]$. The presence of the sub-MHz oscillation infers some intrinsic distortion of the fullerene cages and a precession decoupled from the molecular dynamics. Density functional theory (DFT) simulations of muon and muonium species in different crystalline $\mathrm{C}_{60}$ films [22], identifies the endohedral muonium polaron $\left(\mathrm{ENDO}_{\mathrm{f}}\right)$ state as the energetically most favored product of electron trapping at $\mu^{+}$sites in the presence of free-carriers in the conduction band of the $\mathrm{C}_{60}$ film ([22] Fig. S13 and Table S5), as produced by the $\mu^{+} \mathrm{keV}$ implantation. This polaronic state, with a formation energy of about $-0.72 \mathrm{eV}$, is characterized by a floating geometry for the $\mu^{+}$inside the $\mathrm{C}_{60}\left(\mathrm{ENDO}_{\mathrm{f}}\right)$, slightly offset from the center of mass of the $\mathrm{C}_{60}$ and at a distance of $\sim 3 \AA$ from the closest C-atom ([22] Fig. S7). As shown by the absence of $\mu^{+}-\mathrm{C}$ stretching vibrational modes (Table S3), this geometry presents no chemical bonding between muonium and $\mathrm{C}_{60}$, leading to an electron spin-density localized around the $\mu^{+}$ sites ([22] Fig. S11).

The low-frequency oscillation is due to an axially symmetric hyperfine interaction for $\mathrm{ENDO}_{\mathrm{f}}$. Its frequency depends on film quality, charge state and the local magnetic field. It varies between $0.1 \mathrm{MHz}$ for a highly crystalline, pristine and de- gaussed system, to $0.8 \mathrm{MHz}$ for an amorphous, non-degaussed and/or charged film, e.g., at metallic interfaces. This is in good agreement with the predicted value of $\sim 0.45 \mathrm{MHz}$ for a system with $25 \% \mathrm{C}_{60}$ vacancies (see Table $\mathrm{S} 7$ [22]). Experimentally, the formation energy of the $\mathrm{ENDO}_{\mathrm{f}}$ polaron is $300 \pm 100 \mathrm{meV}$ [Figs. 1(c)-1(d)]. This is a factor two smaller than in the calculations (Table S5 [22]). The difference is probably due to impurities and defects up to $0.9 \mathrm{eV}$ above the top of the valance band [29], limitations in the simulations and the overestimated concentration of $\mu^{+}$in simulations with respect to the experiment, where only one $\mu^{+}$is present in the $\sim 10^{-4} \mathrm{~cm}^{3}$ sample at a time. The overestimated concentration induces unscreened, artificial electrostatic (multipolar) interactions between periodic replicas of the simulation cell that in turn overstabilize the polaronic solution with respect to the delocalized state [Fig. 1(c)].

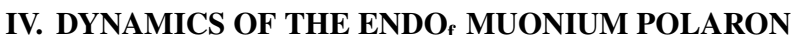

Molecules in $\mathrm{C}_{60}$ films undergo a glass transition at $T_{g} \sim$ $120 \mathrm{~K}$. Below this temperature, the rotational degrees of freedom for the fullerene cages are completely frozen. Above $T_{g}$, a uniaxial ratcheting mechanism between different molecular orientations takes place until around $260 \mathrm{~K}$, where the cages undergo continuous rotational diffusion [31]. Additionally, Born-Oppenheimer, DFT molecular dynamics (BOMD) simulations of different endohedral and exohedral $\mu^{+}$and muonium species confirm the $\mathrm{ENDO}_{\mathrm{f}}$ state as the only system uncorrelated to the $\mathrm{C}_{60}$ rotational dynamics. This makes ENDO $_{\mathrm{f}}$ the only detectable oscillation at $250 \mathrm{~K}$, regardless of the $\mathrm{C}_{60}$ free rotation. Path integral MD (PIMD) simulations of the $\mathrm{ENDO}_{\mathrm{f}}$ state at 50 and $300 \mathrm{~K}$ confirm its localization around, but not exactly at, the center of mass of the $\mathrm{C}_{60}$. There is no quantum tunneling through the $\mathrm{C}_{60}$ cage observed during the (finite) timespan of the simulations [Fig. 2(c)].

The depolarization rate $\lambda$ of the low-frequency oscillation was studied as a function of temperature $T$. We assume $\mathrm{Ar}$ rhenius dynamics, with a decoherence period $\tau$ such that [32]

$$
\lambda \sim \frac{1}{\tau}=A e^{\frac{E_{A}}{k_{B} T}}
$$

where $E_{A}$ is the activation energy, $k_{B}$ is the Boltzmanns constant, and $A$ is a fitting factor. Although the $\mathrm{ENDO}_{\mathrm{f}}$ state appears decoupled from the molecular rotations in the ps scale [see Figs. 2(a)-2(c)], we expect some effect of the ratcheting on the muonium spin direction over the longer decay time scale of $2.2 \mu \mathrm{s}$. The dynamics may have an effect in the temperature-dependent slowing of the decoherence.

The spin depolarization of the $\mathrm{ENDO}_{\mathrm{f}}$ polaron state undergoes thermal changes with characteristic activation energies of the order of $15 \mathrm{meV}$ above $T_{g}$ and $1 \mathrm{meV}$ below $T_{g}$ [Fig. 2(d)]. This observation resembles the temperature dependent, motional line-narrowing experienced in NMR experiments, where the increased motion of a sampling environment due to molecular dynamics causes areas of varying magnetic field to become more homogeneous, reducing the depolarization of the nuclear spin [33,34]. The depolarization above $T_{g}$ is therefore conditioned by the molecular rotations. Below $T_{g}$, the activation energy corresponds to the dynamic barrier for the movement and rotation of the muonium in- 

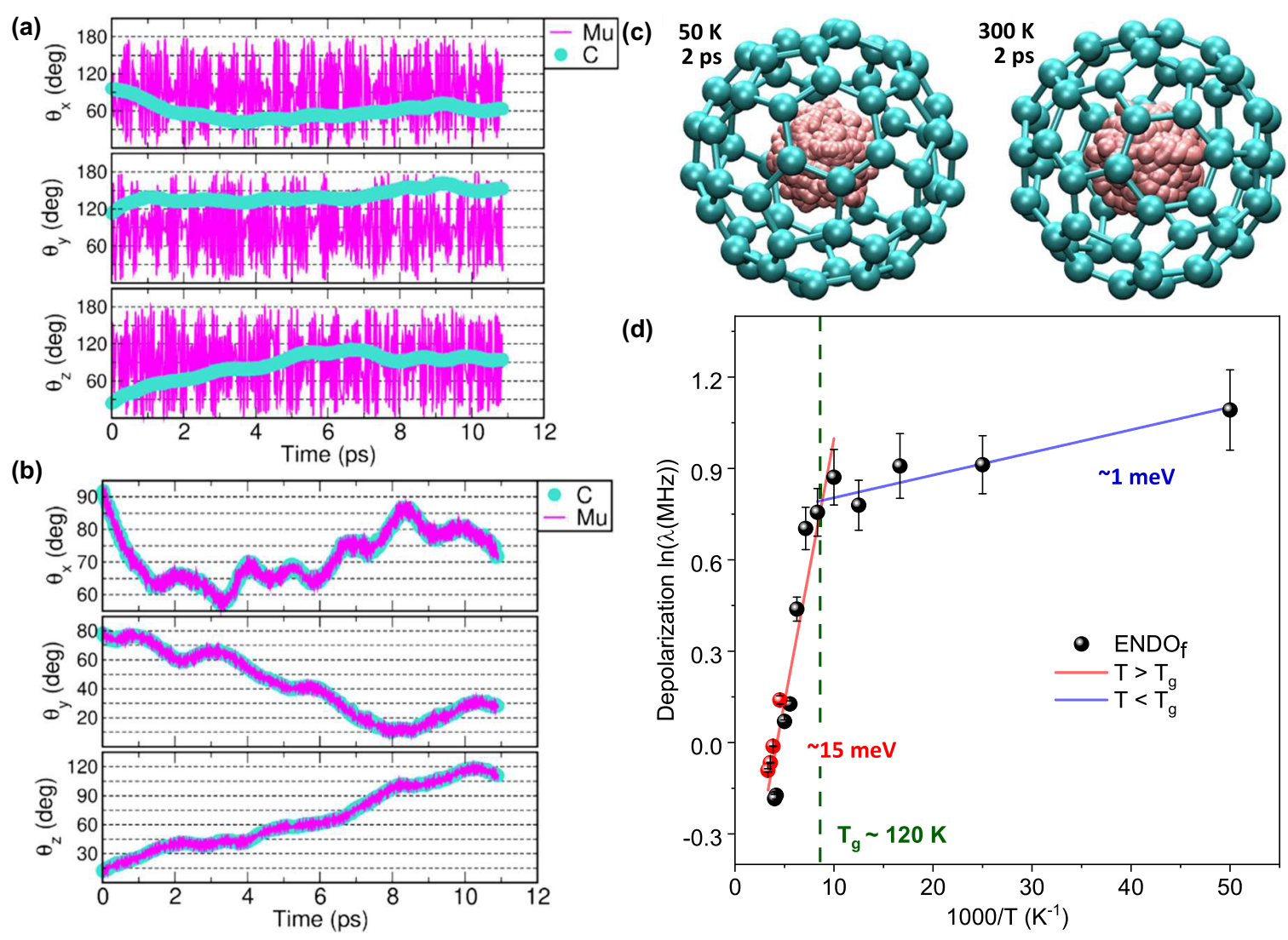

(d)

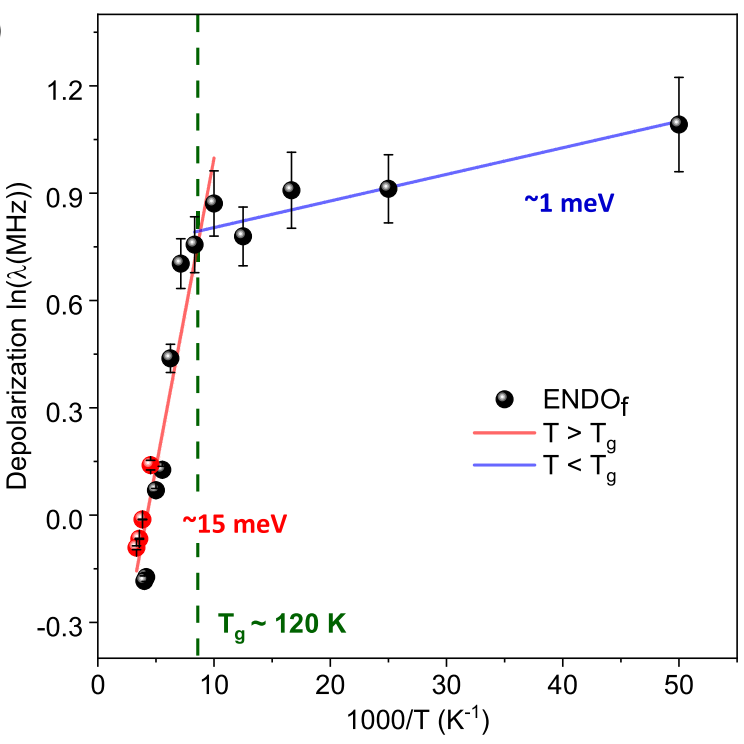

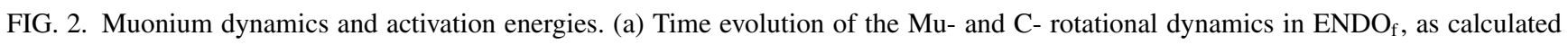
by the angles between the instantaneous $\mathrm{Mu}$ (closest $\mathrm{C}$-atom) radial distance from the $\mathrm{C}_{60}$ 's center of mass and the $\mathrm{x}\left(\theta_{\mathrm{x}}\right), \mathrm{y}\left(\theta_{\mathrm{y}}\right)$ and $\mathrm{z}\left(\theta_{\mathrm{z}}\right)$ axes. The C-trace reports the rotational evolution of the $\mathrm{C}$-atom closest $(\sim 3 \AA)$ to the Mu at the start of the BOMD trajectory. (b) As in a, but for the EXO muonium state. The $\mathrm{C}$-atom closest to the Mu does not change during the whole BOMD trajectory. Note that for the ENDO $\mathrm{f}_{\mathrm{f}}$ state the dynamics of molecule and muonium are decoupled, whereas in the EXO state the muonium follows the molecular rotation. (c) Ab initio path integral molecular dynamics (AI-PIMD) distribution of the muon particle in the $\mathrm{ENDO}_{\mathrm{f}}$ at 50 and $300 \mathrm{~K}$. For each of the 16 beads used in the simulations, 1000 frames ( $2 \mathrm{fs}$ apart) were extracted from the production run and superimposed in the same image. The initial position of the $\mathrm{C}_{60}$ is used to provide a better visualization of the quantum spread of the muonium inside the molecule. C: cyan, Mu: pink. (d) Temperature dependence of the depolarization rate associated with $\mathrm{ENDO}_{\mathrm{f}}$ state, showing the two thermal activation regimes above (red line fit) and below (blue) the glass transition $\mathrm{T}_{\mathrm{g}}$.

side the cage, as observed in both the BOMD (Supplemental Material Figs. S14-S15 [22]) and PIMD [Fig. 2(c)] simulations.

\section{LOW MAGNETIC FIELD DETECTION IN A CRYSTALLINE SAMPLE}

The dependence of the muonium hyperfine transition frequency with a magnetic field was studied using a crystalline $\mathrm{C}_{60}$ film grown at a substrate temperature of $473 \mathrm{~K}$ onto a [110] oriented $\mathrm{Nb}$ layer sputtered at $1073 \mathrm{~K}$ onto $c$-plane $\mathrm{Al}_{2} \mathrm{O}_{3}$ X-ray diffraction spectrum in Fig. S1. When comparing the crystalline $\mathrm{C}_{60}$ to the disordered film grown on $\mathrm{SiO}_{2}$ at room temperature, we note that muonium formation is $30 \%$ of the implanted muons, demonstrating the diamagnetic behavior of the uncoupled $\mu^{+}$particle. The difference suggests a role of the molecular crystal structure to the formation of the muonium polaron, as observed in the calculated vibrational density of states of $\mathrm{C}_{60}$ for crystalline and disordered films. This probably explains the discrepancy between these measurements and past studies on fullerite single crystals. Figures 3 and
Fig. S3 [22] demonstrate that at $250 \mathrm{~K}$, in fields up to $8 \mathrm{G}$, there is only a single frequency line, increasing linearly at $1.402 \pm 0.003 \mathrm{MHz} \mathrm{G}^{-1}$ [35]. This is in line with the standard theoretical predictions for a strongly coupled muon-electron system. Simultaneously, there is axial anisotropy leading to a zero-field frequency of $0.50 \pm 0.02 \mathrm{MHz}$. At $20 \mathrm{~K}$, v $v_{\mathrm{ZF}}$ reduces to $0.15 \pm 0.03 \mathrm{MHz}$ with the slope reducing to $0.74 \pm$ $0.05 \mathrm{MHz} \mathrm{G}^{-1}$ [Fig. 3(b)]. This slope cannot be explained by a simple strong coupling approximation, which predicts the behavior seen in Fig. 3(a). It appears instead in the case of isotropic coupling on the same order of magnitude as the applied field, which suggests a small Fermi contact term for this site; see the Supplemental Material for further detail [22].

\section{PROBING EMERGENT LOCAL MOMENTS AND ELECTRONIC STATES AT METALLOMOLECULAR HETEROSTRUCTURES}

We use the $\mathrm{ENDO}_{\mathrm{f}}$ state to detect small spin-electronic changes at a metallomolecular interface. The sample structure is $\left[\mathrm{Si}(\mathrm{Sub}) / \mathrm{Ta}(3) / \mathrm{Cu}(5) / \mathrm{C}_{60}(114) / \mathrm{Cu}(5) / \mathrm{Au}(14)\right]$, where 

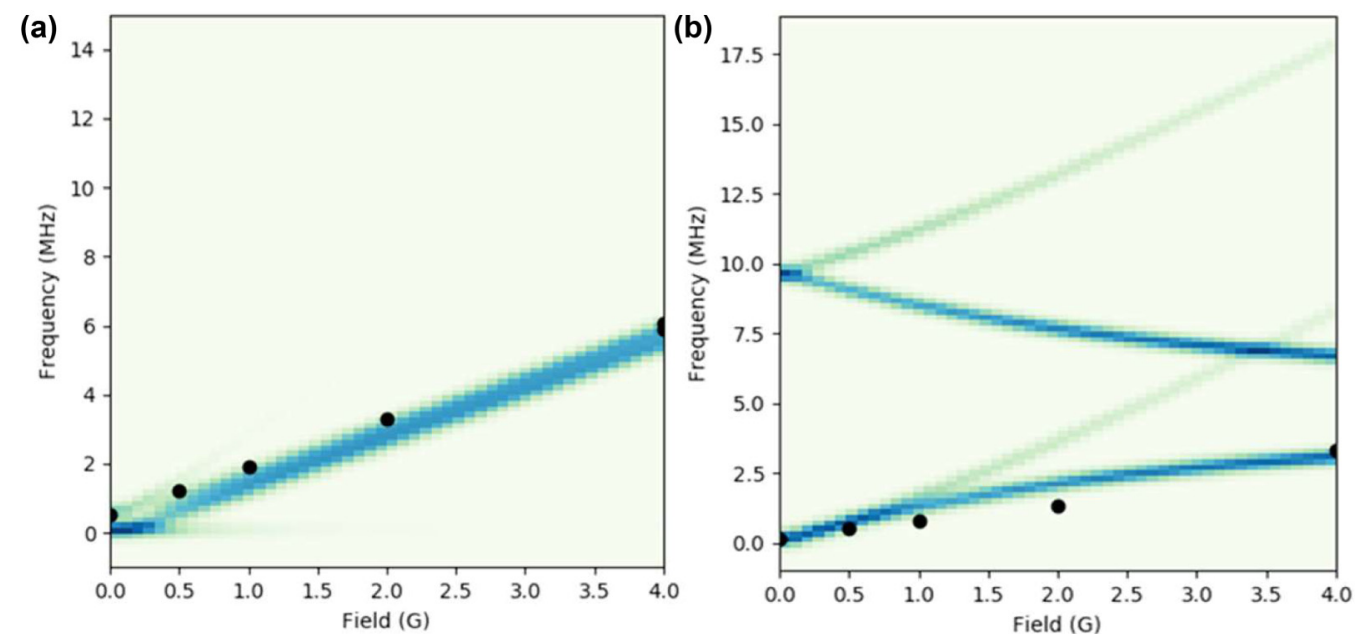

(c)

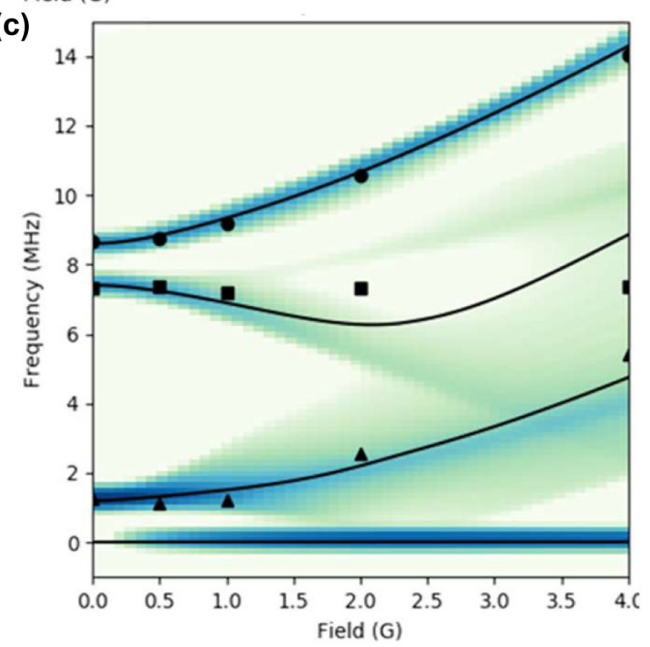

FIG. 3. The magnetic field dependence of the $\mathrm{ENDO}_{\mathrm{f}}$ and EXO muonium oscillation frequencies as measured in the crystalline sample. (a) Observed experimental frequencies (circles) vs simulations (color map) for $\mathrm{ENDO}_{\mathrm{f}}$ at $250 \mathrm{~K}$. (b) $20 \mathrm{~K}$ experiments (circles) and simulations (color map) for the $\mathrm{ENDO}_{\mathrm{f}}$ state, modeled with a hyperfine isotropic coupling $\mathrm{A}_{\text {iso }}=9.65 \mathrm{MHz}$. (c) Observed experimental frequencies (circles, squares, and triangles) vs simulated intensities (color map) for the EXO state. The dipolar part of the tensor was chosen to match the observations at zero field and the Fermi contact term is in the high limit $\left(A_{\text {iso }} \gg 100 \mathrm{MHz}\right)$. Since the middle peaks become very broad and low at high field, the color map here is logarithmic in order to enhance their visibility. This decay with field is observed experimentally [22].

the thicknesses in brackets are in $\mathrm{nm}$. Here, the Ta layer seeds the [111] growth of the $\mathrm{Cu}$ film. The thickness of the $\mathrm{Au}$ layer was chosen to provide adequate moderation of the muon beam, while also protecting the sample against oxidation. Measurements were taken at $250 \mathrm{~K}$, where the time scale of the rotation of the molecule is short enough that the EXO muonium does not contribute to the observed signal. The sample was grown in a forming field of $20 \mathrm{mT}$. The sample was first measured in ZF before any other magnetic field was applied. The time-dependent polarization of the muonium state in ZF has been fitted with a single frequency model with the addition of an $A_{\text {tail }}$ decay term which accounts for the slow depolarization of diamagnetic $\mu^{+}$particles within the metal layers:

$$
P(t)=A_{M u} \cos \left(2 \pi v_{M u} t\right) e^{\left(-\lambda_{M u} t\right)}+A_{\text {tail }} e^{\left(-\lambda_{\text {tail }} t\right)} .
$$

This yields the $\mathrm{ENDO}_{\mathrm{f}}$ oscillation frequency, $v_{\mathrm{Mu}}$. The depolarization rate, $\lambda_{\mathrm{Mu}}$, is also obtained. $\lambda_{\mathrm{Mu}}$ characterizes the distribution of hyperfine oscillations contributing towards the signal at any given implantation energy. Figure 4(b) shows the dependence of the percentage change in $\mathrm{ENDO}_{\mathrm{f}}$ frequency, $\Delta v_{\mathrm{Mu}}$, as a function of implantation depth. The average implantation depth has been calculated from the simulated stopping profiles shown in Fig. 4(a). $\Delta v_{\mathrm{Mu}}$ has been calculated relative to the frequency at $12 \mathrm{keV}$; the implantation energy that captures the center-most region. In the initial, asgrown measurement, a curved profile of muonium frequency is observed with depth. $v_{\mathrm{Mu}}$ is higher at the top and bottom interfaces by $14 \pm 5 \%$ and $25 \pm 5 \%$, respectively. From this measurement alone, it is clear that towards the $\mathrm{C}_{60} / \mathrm{Cu}$ interfaces, there is a change in the hyperfine splitting between $\mathrm{ENDO}_{\mathrm{f}}$ spin configurations. Orbital rehybridization, lattice reconstruction, and Fermi level matching, lead to chargetransfer from the metal into the first fullerene monolayer [36-39]. As the neutral $\mathrm{C}_{60}$ lowest unoccupied molecular orbital is triply degenerate, the addition of charge is accompanied by a molecular distortion to break the degeneracy through the Jahn-Teller effect [40]. The reduced symmetry due to the molecular distortion imparts anisotropy onto the hyperfine 
(a)
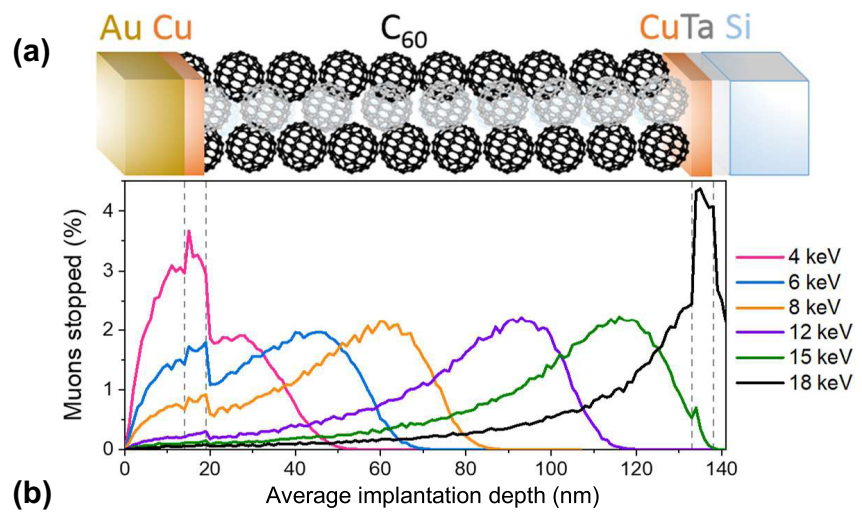

(b)

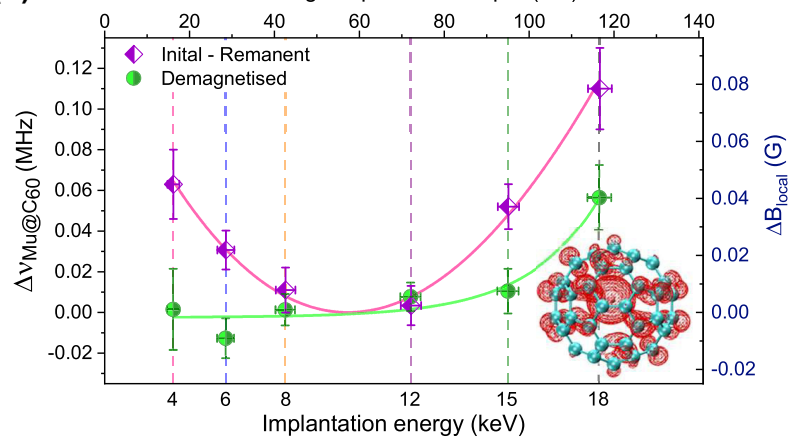

FIG. 4. ZF-LE- $\mu$ SR measurements of the $\mathrm{Mu} @ \mathrm{C}_{60}$ frequency, $v_{\mathrm{Mu}}$, and depth profile in a thin film heterostructure. (a) The simulated implantation profile for a structure of $\left[\mathrm{Si}(\mathrm{Sub}) / \mathrm{Ta}(3) / \mathrm{Cu}(5) / \mathrm{C}_{60}(114) / \quad \mathrm{Cu}(5) / \mathrm{Au}(14)\right]$, where the thicknesses in brackets are in $\mathrm{nm}$. At the 4 and $18 \mathrm{keV}$ implantation energies, a considerable percentage (57\% and $34 \%$, respectively) of muons stop in the metal layers. As the measurement was performed in $\mathrm{ZF}$, these are not expected to contribute to the oscillating signal. (b) The obtained depth dependence of the relative change in the $\mathrm{ENDO}_{\mathrm{f}}$ oscillation frequency, $\Delta v_{\mathrm{Mu}}$, for the initial state measurement and following a degauss; lines are a guide for the eye. The corresponding change in the local field, $\Delta \mathrm{B}_{\text {Local }}$, calculated from the measured calibration curve in the crystalline $\mathrm{C}_{60}$ sample is also shown. Inset shows the highly anisotropic spin density (red: $10^{-6} \mu_{\mathrm{B}} \AA^{-3}$ ) for the endohedral muonium polaron state with an additional electron $\mathrm{ENDO}_{\mathrm{f}}(-)$ calculated using screened-hybrid DFT (HSE06, see Supplemental Material).

coupling tensor, which describes the energy splitting in zerofield of the triplet muonium spin configurations. This is the cause of the observable $0.7 \mathrm{MHz} \mathrm{ZF}$ oscillation in the oblate molecule, $\mathrm{C}_{70}[9,10,41]$.

To examine the origin of the frequency shifts at the metallomolecular interfaces, we compare the as-grown state with the signal after the sample is degaussed in a damped alternating field starting at $300 \mathrm{G}$. Following this degauss procedure, the depth profile of the muonium frequency dramatically changes. As shown in Fig. 4(b), $v_{\mathrm{Mu}}$ now remains almost constant throughout the molecular layer. The increased $v_{\mathrm{Mu}}$ frequency at both metal interfaces before degauss is therefore a consequence of Zeeman splitting of the muonium hyperfine levels due to small stray fields, of the order of $20-80 \mathrm{mG}$, originating at the $\mathrm{Cu} / \mathrm{C}_{60}$ interface. After degauss, the remaining $9 \pm 3 \%$ frequency shift at the depth closest to the bottom $\mathrm{Cu} / \mathrm{C}_{60}$ interface can be attributed to metal-to-molecule electron trans- fer at the interface and the presence of negatively charged $\mathrm{ENDO}_{\mathrm{f}}(-)$. This state has a more anisotropic spin density, leading to higher oscillation frequency [inset in Fig. 4(b) and Supplemental Material Fig. S11]. The fact that there is no change in $v_{\mathrm{Mu}}$ at the top $\mathrm{Cu} / \mathrm{C}_{60}$ interface after degauss suggests a lower coupling and damage of the molecular layer with the metal layer sputtered on top as compared to the bottom interface, where the molecules were evaporated on the metal.

\section{CONCLUSION}

We have presented LE- $\mu$ SR data that characterizes the dynamics of the endohedral floating muonium polaronic state in $\mathrm{C}_{60}$ layers. The hyperfine frequencies of this state map the emergent magnetism formed at a $\mathrm{Cu} / \mathrm{C}_{60}$ interface via the Zeeman splitting of the muonium hyperfine coupling. DFT calculations were used to model the charge, spin density, and dynamics of this muonium state, confirming it as the only system being detectable for temperatures $>260 \mathrm{~K}$ as observed in the experiments. The polaron state causes a small anisotropy in the hyperfine coupling of the endohedral muonium, which allows us to observe a $\mathrm{ZF}$ precession of a few hundred $\mathrm{kHz}$. This precession is very sensitive to any deviation from $\mathrm{ZF}$ and therefore is highly sensitive to weak stray magnetic fields of weak or dilute magnetic moments. For a nonpolaronic, isotropic muonium state, to measure a precession we need to apply a magnetic field. X-ray-based synchrotron techniques have been used to observe magnetic moments in $\mathrm{Cu}$ as small as $10^{-5} \mu_{\mathrm{B}}$, which is roughly equivalent to local fields of $0.1 \mathrm{G}$ $[5,6]$. The $\mathrm{ENDO}_{\mathrm{f}}$ polaronic state can detect local magnetic fields of $\sim 0.01$ Gauss and the charge transfer at a metallomolecular interface [Fig. 4(b)]. When X-ray techniques are element sensitive, the muonium polaron offers the possibility to map the depth profile of the magnetization and the distribution of free charge in, e.g., magnetic multilayers, hybrid batteries or photovoltaic devices. $\mathrm{C}_{60}$ or other compounds forming muon polaron states such as crystalline semiconductors or oxides [42] are part of, or can be added, to the device structure $[7,8]$. For example, fullerene layers can be evaporated in situ on top of candidates to two-dimensional magnetism or ferroelectric materials to study weak surface effects such as magnetic monopoles [14].

\section{ACKNOWLEDGMENTS}

We thank the Engineering and Physical Sciences Research Council (EPSRC UK) for support via Grants No. EP/M000923/1, No. EP/K036408/1, No. EP/I004483/1, No. EP/S031081/1, and No. EP/S030263/1. L.L., S.S., D.J. and G.T. acknowledge also support from STFC-ISIS Neutron and Muon Source and Ada Lovelace Centre at STFC-SCD. We acknowledge use of the ARCHER (via the U.K. Car-Parrinello Consortium, EP/P022618/1 and EP/P022189/2), U.K. Materials and Molecular Modelling Hub (EP/P020194/1), and STFC Scientific Computing Department's SCARF HCP facilities. The LE- $\mu \mathrm{SR}$ measurements were performed at the Swiss Muon Source $\mathrm{S} \mu \mathrm{S}$, Paul Scherrer Institute, Villigen, Switzerland. We acknowledge support from the Henry Royce Institute. This work was also supported financially through the EPSRC Grant Nos. EP/ P022464/1, and EP/R00661X/1. 
[1] C. Barraud, P. Seneor, R. Mattana, S. Fusil, K. Bouzehouane, C. Deranlot, P. Graziosi, L. Hueso, I. Bergenti, V. Dediu, F. Petroff, and A. Fert, Unravelling the role of the interface for spin injection into organic semiconductors, Nat. Phys. 6, 615 (2010).

[2] M. Cinchetti, V. A. Dediu, and L. E. Hueso, Activating the molecular spinterface, Nat. Mater. 16, 507 (2017).

[3] F. Al Ma'Mari, T. Moorsom, G. Teobaldi, W. Deacon, T. Prokscha, H. Luetkens, S. Lee, G. E. Sterbinsky, D. A. Arena, D. A. MacLaren, M. Flokstra, M. Ali, M. C. Wheeler, G. Burnell, B. J. Hickey, and O. Cespedes, Beating the Stoner criterion using molecular interfaces, Nature (London) 524, 69 (2015).

[4] F. Al Ma'Mari, M. Rogers, S. Alghamdi, T. Moorsom, S. Lee, T. Prokscha, H. Luetkens, M. Valvidares, G. Teobaldi, M. Flokstra, R. Stewart, P. Gargiani, M. Ali, G. Burnell, B. J. Hickey, and O. Cespedes, Emergent magnetism at transitionmetal-nanocarbon interfaces, Proc. Natl. Acad. Sci. USA 114, 5583 (2017).

[5] J. Li, L. R. Shelford, P. Shafer, A. Tan, J. X. Deng, P. S. Keatley, C. Hwang, E. Arenholz, G. van der Laan, R. J. Hicken, and Z. Q. Qiu, Direct Detection of Pure ac Spin Current by X-Ray PumpProbe Measurements, Phys. Rev. Lett. 117, 076602 (2016).

[6] R. Kukreja, S. Bonetti, Z. Chen, D. Backes, Y. Acremann, J. A. Katine, A. D. Kent, H. A. Durr, H. Ohldag, and J. Stohr, X-Ray Detection of Transient Magnetic Moments Induced by a Spin Current in Cu, Phys. Rev. Lett. 115, 096601 (2015).

[7] Y. H. Xiao, Y. Wang, S. H. Bo, J. C. Kim, L. J. Miara, and G. Ceder, Understanding interface stability in solid-state batteries, Nat. Rev. Mater. 5, 105 (2020).

[8] T. Gatti, E. Menna, M. Meneghetti, M. Maggini, A. Petrozza, and F. Lamberti, The Renaissance of fullerenes with perovskite solar cells, Nano Energy 41, 84 (2017).

[9] U. Binninger, C. Bernhard, A. Hofer, C. Niedermayer, E. Recknagel, J. Erxmeyer, and A. Weidinger, Rotationaldynamics of solid c-70 investigated by the muon-spin-rotation technique, Phys. Rev. B 51, 14867 (1995).

[10] K. Prassides, T. J. S. Dennis, C. Christides, E. Roduner, H. W. Kroto, R. Taylor, and D. R. M. Walton, MU-at-the-cost-of- $\mathrm{C}_{70^{-}}$ monitoring the dynamics of fullerenes from inside the cage, J. Phys. Chem. 96, 10600 (1992).

[11] R. F. Kiefl, T. L. Duty, J. W. Schneider, A. Macfarlane, K. Chow, J. W. Elzey, P. Mendels, G. D. Morris, J. H. Brewer, E. J. Ansaldo, C. Niedermayer, D. R. Noakes, C. E. Stronach, B. Hitti, and J. E. Fischer, Evidence for Endohedral Muonium In $\mathrm{K}_{x} \mathrm{C}_{60}$ And Consequences For Electronic-Structure, Phys. Rev. Lett. 69, 2005 (1992).

[12] A. Lappas, K. Prassides, K. Vavekis, D. Arcon, R. Blinc, P. Cevc, A. Amato, R. Feyerherm, F. N. Gygax, and A. Schenck, Spontaneous magnetic-ordering in the fullerene charge-transfer salt (tdae) $\mathrm{C}_{60}$, Science 267, 1799 (1995).

[13] A. J. Drew, J. Hoppler, L. Schulz, F. L. Pratt, P. Desai, P. Shakya, T. Kreouzis, W. P. Gillin, A. Suter, N. A. Morley, V. K. Malik, A. Dubroka, K. W. Kim, H. Bouyanfif, F. Bourqui, C. Bernhard, R. Scheuermann, G. J. Nieuwenhuys, T. Prokscha, and E. Morenzoni, Direct measurement of the electronic spin diffusion length in a fully functional organic spin valve by low-energy muon spin rotation, Nat. Mater. 8, 109 (2009).

[14] Q. N. Meier, M. Fechner, T. Nozaki, M. Sahashi, Z. Salman, T. Prokscha, A. Suter, P. Schoenherr, M. Lilienblum, P. Borisov,
I. E. Dzyaloshinskii, M. Fiebig, H. Luetkens, and N. A. Spaldin, Search for the Magnetic Monopole at a Magnetoelectric Surface, Phys. Rev. X 9, 011011 (2019).

[15] A. Di Bernardo, Z. Salman, X. L. Wang, M. Amado, M. Egilmez, M. G. Flokstra, A. Suter, S. L. Lee, J. H. Zhao, T. Prokscha, E. Morenzoni, M. G. Blamire, J. Linder, and J. W. A. Robinson, Intrinsic Paramagnetic Meissner Effect Due to s-Wave Odd-Frequency Superconductivity, Phys. Rev. X 5, 041021 (2015).

[16] M. G. Flokstra, R. Stewart, N. Satchell, G. Burnell, H. Luetkens, T. Prokscha, A. Suter, E. Morenzoni, S. Langridge, and S. L. Lee, Observation of Anomalous Meissner Screening in $\mathrm{Cu} / \mathrm{Nb}$ and $\mathrm{Cu} / \mathrm{Nb} / \mathrm{Co}$ Thin Films, Phys. Rev. Lett. 120, 247001 (2018).

[17] T. Prokscha, E. Morenzoni, C. David, A. Hofer, H. Gluckler, and L. Scandella, Moderator gratings for the generation of epithermal positive muons, Appl. Surf. Sci. 172, 235 (2001).

[18] T. Prokscha, E. Morenzoni, K. Deiters, F. Foroughi, D. George, R. Kobler, A. Suter, and V. Vrankovic, The new mu E4 beam at PSI: A hybrid-type large acceptance channel for the generation of a high intensity surface-muon beam, Nucl. Instrum. Methods Phys. Res. 595, 317 (2008).

[19] E. Morenzoni, H. Glucker, T. Prokscha, R. Khasanov, H. Luetkens, M. Birke, E. M. Forgan, C. Niedermayer, and M. Pleines, Implantation studies of $\mathrm{keV}$ positive muons in thin metallic layers, Nucl. Instrum. Meth. B 192, 254 (2002).

[20] R. M. Macrae, K. Prassides, I. M. Thomas, E. Roduner, C. Niedermayer, U. Binninger, C. Bernhard, A. Hofer, and I. D. Reid, Reorientational dynamics of solid c-70 probed by positive muons, J. Phys. Chem. 98, 12133 (1994).

[21] A. Suter and B. M. Wojek, Musrfit: A free platformindependent framework for $\mu$ SR data analysis, Phys. Proc. 30, 69 (2012).

[22] See Supplemental Material at http://link.aps.org/supplemental/ 10.1103/PhysRevB.104.064429 for for further details on sample structure, low energy muon spin spectroscopy measurements, simulation of spectral intensities and DFT methods, which includes Refs. [17-19,43-76].

[23] W. I. F. David, R. M. Ibberson, T. J. S. Dennis, J. P. Hare, and K. Prassides, Structural phase-transitions in the fullerene $\mathrm{C}_{60}$, Europhys. Lett. 18, 219 (1992).

[24] P. A. Heiney, J. E. Fischer, A. R. McGhie, W. J. Romanow, A. M. Denenstein, J. P. McCauley, A. B. Smith, and D. E. Cox, Orientational Ordering Transition in Solid $\mathrm{C}_{60}$, Phys. Rev. Lett. 66, 2911 (1991).

[25] M. S. Dresselhaus, G. Dresselhaus, and P. C. Eklund, Science of Fullerenes and Carbon Nanotubes (Academic Press, New York, 1996).

[26] T. L. Duty, J. H. Brewer, K. Chow, R. F. Kiefl, A. W. Macfarlane, G. D. Morris, J. W. Schneider, B. Hitti, R. Lichti, L. Brard, J. E. Fischer, A. B. Smith, and R. M. Strongin, Zero-field mu-sr in crystalline $\mathrm{C}_{60}$, Hyperfine Interact. 86, 789 (1994).

[27] R. F. Kiefl, J. W. Schneider, A. Macfarlane, K. Chow, T. L. Duty, T. L. Estle, B. Hitti, R. L. Lichti, E. J. Ansaldo, C. Schwab, P. W. Percival, G. Wei, S. Wlodek, K. Kojima, W. J. Romanow, J. P. McCauley, N. Coustel, J. E. Fischer, and A. B. Smith, Molecular-Dynamics of the Muonium- $\mathrm{C}_{60}$ Radical In Solid C60, Phys. Rev. Lett. 68, 2708(E) (1992).

[28] E. J. Ansaldo, C. Niedermayer, and C. E. Stronach, Muonium in fullerite, Nature (London) 353, 121 (1991). 
[29] S. Gonda, M. Kawasaki, T. Arakane, and H. Koinuma, in Novel Forms of Carbon II, edited by C. L. Renschler et al., MRS Symposium Proceedings (Cambridge University Press, Cambridge, 1994), pp. 325.

[30] Z. Salman, T. Prokscha, A. Amato, E. Morenzoni, R. Scheuermann, K. Sedlak, and A. Suter, Direct Spectroscopic Observation of a Shallow Hydrogenlike Donor State in Insulating $\mathrm{SrTiO}_{3}$, Phys. Rev. Lett. 113, 156801 (2014).

[31] E. Abou-Hamad, Y. Kim, T. Wagberg, D. Boesch, S. Aloni, A. Zettl, A. Rubio, D. E. Luzzi, and C. Goze-Bac, Molecular dynamics and phase transition in one-dimensional crystal of c-60 encapsulated inside single wall carbon nanotubes, ACS Nano 3, 3878 (2009).

[32] I. McKenzie and S. P. Cottrell, Microscopic muon dynamics in the polymer electrolyte poly(ethylene oxide), Phys. Rev. E 96, 012502 (2017).

[33] P. Jain, A. Levchenko, P. Yu, and S. Sen, Molecular dynamics in supercooled glycerol: Results from C-13 NMR spectroscopy, J. Chem. Phys. 130, 194506 (2009).

[34] S. Adhikari, M. Selmke, and F. Cichos, Temperature dependent single molecule rotational dynamics in PMA, Phys. Chem. Chem. Phys. 13, 1849 (2011).

[35] B. D. Patterson, Muonium states in semiconductors, Rev. Mod. Phys. 60, 69 (1988).

[36] J. A. Larsson, S. D. Elliott, J. C. Greer, J. Repp, G. Meyer, and R. Allenspach, Orientation of individual $\mathrm{C}_{60}$ molecules adsorbed on $\mathrm{Cu}(111)$ : Low-temperature scanning tunneling microscopy and density functional calculations, Phys. Rev. B 77, 115434 (2008).

[37] W. W. Pai, H. T. Jeng, C. M. Cheng, C. H. Lin, X. D. Xiao, A. D. Zhao, X. Q. Zhang, G. Xu, X. Q. Shi, M. A. Van Hove, C. S. Hsue, and K. D. Tsuei, Optimal Electron Doping of a $\mathrm{C}_{60}$ Monolayer on $\mathrm{Cu}(111)$ via Interface Reconstruction, Phys. Rev. Lett. 104, 036103 (2010).

[38] G. Xu, X. Q. Shi, R. Q. Zhang, W. W. Pai, H. T. Jeng, and M. A. Van Hove, Detailed low-energy electron diffraction analysis of the $(4 \times 4)$ surface structure of $\mathrm{C}_{60}$ on $\mathrm{Cu}(111)$ : Seven-atomvacancy reconstruction, Phys. Rev. B 86, 075419 (2012).

[39] L. Martin-Olivera, D. G. Shchukin, and G. Teobaldi, Role of metal lattice expansion and molecular pi-conjugation for the magnetic hardening at $\mathrm{Cu}$-organics interfaces, J. Phys. Chem. C 121, 23777 (2017).

[40] C. C. Chancey and M. C. M. O'Brien, The Jahn-Teller Effect in $\mathrm{C}_{60}$ and Other Icosahedral Complexes (Princeton University Press, Princeton, NJ, 1997), p. 97.

[41] C. Niedermayer, Fullerenes with $\mu \mathrm{SR}$, Hyperfine Interact. 97, 285 (1996).

[42] M. H. Dehn, J. K. Shenton, S. Holenstein, Q. N. Meier, D. J. Arseneau, D. L. Cortie, B. Hitti, A. C. Y. Fang, W. A. MacFarlane, R. M. L. McFadden, G. D. Morris, Z. Salman, H. Luetkens, N. A. Spaldin, M. Fechner, and R. F. Kiefl, Observation of a Charge-Neutral Muon-Polaron Complex in Antiferromagnetic $\mathrm{Cr}_{2} \mathrm{O}_{3}$, Phys. Rev. X 10, 011036 (2020).

[43] T. Moorsom, M. Rogers, I. Scivetti, S. Bandaru, G. Teobaldi, M. Valvidares, M. Flokstra, S. Lee, R. Stewart, T. Prokscha, P. Gargiani, N. Alosaimi, G. Stefanou, M. Ali, F. Al Ma'Mari, G. Burnell, B. J. Hickey, and O. Cespedes, Reversible spin storage in metal oxide-fullerene heterojunctions, Sci. Adv. 6, eaax1085 (2020).
[44] J. S. Lord, Computer simulation of muon spin evolution, Physica B 374, 472 (2006).

[45] S. K. Zaremba, Good lattice points, discrepancy, and numerical integration, Ann. Mater. Pura Appl. 73, 293 (1966).

[46] H. Conroy, Molecular Schrodinger equation.VIII. A new method for evaluation of multidimensional integrals, J. Chem. Phys. 47, 5307 (1967).

[47] V. B. Cheng, H. H. Suzukawa, and M. Wolfsberg, Investigations of a nonrandom numerical-method for multidimensional integration, J. Chem. Phys. 59, 3992 (1973).

[48] G. Kresse and J. Furthmuller, Efficient iterative schemes for ab initio total-energy calculations using a plane-wave basis set, Phys. Rev. B 54, 11169 (1996).

[49] J. P. Perdew, K. Burke, and Y. Wang, Generalized gradient approximation for the exchange-correlation hole of a manyelectron system, Phys. Rev. B 54, 16533 (1996).

[50] S. Grimme, Semiempirical GGA-type density functional constructed with a long-range dispersion correction, J. Comput. Chem. 27, 1787 (2006).

[51] G. Henkelman, B. P. Uberuaga, and H. Jonsson, A climbing image nudged elastic band method for finding saddle points and minimum energy paths, J. Chem. Phys. 113, 9901 (2000).

[52] G. Henkelman and H. Jonsson, Improved tangent estimate in the nudged elastic band method for finding minimum energy paths and saddle points, J. Chem. Phys. 113, 9978 (2000).

[53] A. V. Krukau, O. A. Vydrov, A. F. Izmaylov, and G. E. Scuseria, Influence of the exchange screening parameter on the performance of screened hybrid functionals, J. Chem. Phys. 125, 224106 (2006).

[54] C. G. Van de Walle and J. Neugebauer, First-principles calculations for defects and impurities: Applications to III-nitrides, J. Appl. Phys. 95, 3851 (2004).

[55] C. Freysoldt, B. Grabowski, T. Hickel, J. Neugebauer, G. Kresse, A. Janotti, and G C., Van de Walle, First-principles calculations for point defects in solids, Rev. Mod. Phys. 86, 253 (2014).

[56] E. Y. Kolyadina, L. A. Matveeva, P. L. Neluba, and E. F. Venger, Analysis of the fundamental absorption edge of the films obtained from the $\mathrm{C}_{60}$ fullerene molecular beam in vacuum and effect of internal mechanical stresses on it, Semicond. Phys. Quantum Electron. 18, 349 (2015).

[57] T. U. Ito, W. Higemoto, A. Koda, and K. Shimomura, Polaronic nature of a muonium-related paramagnetic center in $\mathrm{SrTiO}_{3}$ Appl. Phys. Lett. 115, 192103 (2019).

[58] M. Reticcioli, M. Setvin, X. Hao, P. Flauger, G. Kresse, M. Schmid, U. Diebold, and C. Franchini, Polaron-Driven Surface Reconstructions, Phys. Rev. X 7, 031053 (2017).

[59] T. D. Kuhne, M. Iannuzzi, M. Del Ben, V. V. Rybkin, P. Seewald, F. Stein, T. Laino, R. Z. Khaliullin, O. Schutt, F. Schiffmann, D. Golze, J. Wilhelm, S. Chulkov, M. H. BaniHashemian, V. Weber, U. Borstnik, M. Taillefumier, A. S. Jakobovits, A. Lazzaro, H. Pabst, T. Muller, R. Schade, M. Guidon, S. Andermatt, N. Holmberg, G. K. Schenter, A. Hehn, A. Bussy, F. Belleflamme, G. Tabacchi, A. Gloss, M. Lass, I. Bethune, C. J. Mundy, C. Plessl, M. Watkins, J. VandeVondele, M. Krack, and J. Hutter, CP2K: An electronic structure and molecular dynamics software package - Quickstep: Efficient and accurate electronic structure calculations, J. Chem. Phys. 152, 194103 (2020). 
[60] S. Goedecker, M. Teter, and J. Hutter, Separable dual-space Gaussian pseudopotentials, Phys. Rev. B 54, 1703 (1996).

[61] J. VandeVondele and J. Hutter, Gaussian basis sets for accurate calculations on molecular systems in gas and condensed phases, J. Chem. Phys. 127, 114105 (2007).

[62] S. Grimme, J. Antony, S. Ehrlich, and H. Krieg, A consistent and accurate $a b$ initio parametrization of density functional dispersion correction (DFT-D) for the 94 elements H-Pu, J. Chem. Phys. 132, 154104 (2010).

[63] D. Marx and M. Parrinello, Ab initio path integral molecular dynamics: Basic ideas, J. Chem. Phys. 104, 4077 (1996).

[64] M. Ceriotti and D. E. Manolopoulos, Efficient First-Principles Calculation of the Quantum Kinetic Energy and Momentum Distribution of Nuclei, Phys. Rev. Lett. 109, 100604 (2012).

[65] F. Uhl, D. Marx, and M. Ceriotti, Accelerated path integral methods for atomistic simulations at ultra-low temperatures, J. Chem. Phys. 145, 054101 (2016).

[66] Y. Nagata, R. E. Pool, E. H. G. Backus, and M. Bonn, Nuclear Quantum Effects Affect Bond Orientation of Water at the Water-Vapor Interface, Phys. Rev. Lett. 109, 226101 (2012).

[67] O. Marsalek and T. E. Markland, Ab initio molecular dynamics with nuclear quantum effects at classical cost: Ring polymer contraction for density functional theory, J. Chem. Phys. 144, 054112 (2016).

[68] C. Schran, F. Brieuc, and D. Marx, Converged colored noise path integral molecular dynamics study of the zundel cation down to ultralow temperatures at coupled cluster accuracy, J. Chem. Theory Comput. 14, 5068 (2018).
[69] S. J. Clark, M. D. Segall, C. J. Pickard, P. J. Hasnip, M. J. Probert, K. Refson, and M. C. Payne, First-principles methods using CASTEP, Z. Kristallogr. 220, 567 (2005).

[70] J. P. Perdew, K. Burke, and M. Ernzerhof, Generalized Gradient Approximation Made Simple, Phys. Rev. Lett. 78, 1396 (1997).

[71] J. S. Lin, A. Qteish, M. C. Payne, and V. Heine, Optimized and transferable nonlocal separable ab initio pseudopotentials, Phys. Rev. B 47, 4174 (1993).

[72] H. J. Monkhorst and J. D. Pack, Special points for Brillouinzone integrations, Phys. Rev. B 13, 5188 (1976).

[73] B. S. Hudson and S. K. Chafetz, Zero-point corrections for isotropic coupling constants for cyclohexadienyl radical, $\mathrm{C} 6 \mathrm{H} 7$ and $\mathrm{C}(6) \mathrm{H}(6) \mathrm{Mu}$ : Beyond the bond length change approximation, Molecules 18, 4906 (2013).

[74] I. McKenzie, J. C. Brodovitch, P. W. Percival, T. Ramnial, and J. A. C. Clyburne, The reactions of imidazol-2-ylidenes with the hydrogen atom: A theoretical study and experimental confirmation with muonium, J. Am. Chem. Soc. 125, 11565 (2003).

[75] C. H. Yue, L. Liborio, T. Bian, S. Sturniolo, J. Wright, S. P. Cottrell, R. Khasanov, G. Simutis, U. A. Jayasooriya, and Y. Chao, A Muon Spectroscopic and Computational Study of the Microscopic Electronic Structure in Thermoelectric Hybrid Silicon Nanostructures, J. Phys. Chem. C 124, 9656 (2020).

[76] J. S. Moller, D. Ceresoli, T. Lancaster, N. Marzari, and S. J. Blundell, Quantum states of muons in fluorides, Phys. Rev. B 87, 121108(R) (2013). 